\title{
The Future Role of High-Performance Computing in Cardiovascular Medicine and Science -Impact of Multi-Dimensional Data Analysis-
}

\author{
Shinya Goto ${ }^{1}$, Darren K. McGuire ${ }^{2}$ and Shinichi Goto ${ }^{1}$
}

${ }^{1}$ Department of Medicine (Cardiology), Tokai University School of Medicine, Isehara, Japan

${ }^{2}$ Department of Internal Medicine, Division of Cardiology University of Texas Southwestern Medical Center and Parkland Health and Hospital System, Dallas, Texas, US

Advances in High-performance computing (HPC) technology have reached the capacity to inform cardiovascular (CV) science in the realm of both inductive and constructive approaches. Clinical trials allow for the comparison of the effect of an intervention without the need to understand the mechanism. This is a typical example of an inductive approach. In the HPC field, training an artificial intelligence (AI) model, constructed by neural networks, to predict future $\mathrm{CV}$ events with the use of large scale multi-dimensional datasets is the counterpart that may rely on as well as inform understanding of mechanistic underpinnings for optimization. However, in contrast to clinical trials, AI can calculate event risk at the individual level and has the potential to inform and refine the application of personalized medicine.

Despite this clear strength, results from AI analyses may identify otherwise unidentified/unexpected (i.e. non-intuitive) relationships between multi-dimensional data and clinical outcomes that may further unravel potential mechanistic pathways and identify potential therapeutic targets, therebycontributing to the parsing of observational associations from causal links. The constructive approach will remain critical to overcome limitations of existing knowledge and anchored biases to actualize a more sophisticated understanding of the complex pathobiology of CV diseases.

HPC technology has the potential to underpin this constructive approach in CV basic and clinical science. In general, even complex biological phenomena can be reduced to combinations of simple biological/chemical/ physical laws. In the deductive approach, the focus/intent is to explain complex CV diseases by combinations of simple principles.

Key words: HPC, AI, MD, Molecular dinamics, Artificial intelligence, Neural network, Machine learning

\section{The Potential Role of High-Performance Computing in Cardiovascular Science}

High-performance computing (HPC) refers to the technology that capitalizes on computing power to deliver high-performance solutions to address major challenges and limitations in engineering, business, science, and medicine, with ever increasing potential across disciplines as data handling and computational capacity continue to expand ${ }^{1,2)}$. The capability of HPC for handling huge volumes of data yields substantial possible utility and application in cardiovascular (CV) science and practice. One promising example of HPC with potential clinical utility is the use of artificial intelligence (AI) constructed by neural networks ${ }^{3)}$. This technology provides the ability to detect quantitative relationships between multi-dimensional data, such as digital images and videos; time-sequential biomarker data; and multiple demographics and single dimensional clinical outcomes (Fig. 1). While the human brain can process the associations between multi-dimensional data and single dimensional outcomes at some level for medical decision making, human decisions are confounded by limited objective analytic capability across multiple dimensions, biases based on

Address for correspondence: Shinya Goto, Department of Medicine (Cardiology), Tokai University School of Medicine, 143 Shimokasuya, Isehara, Kangawa 2591193, Japan E-mail: sgoto3@mac.com

Received: September 8, 2021 Accepted for publication: September 13, 2021

Copyright@2021 Japan Atherosclerosis Society

This article is distributed under the terms of the latest version of CC BY-NC-SA defined by the Creative Commons Attribution License.

\section{Advance Publication Journal of Atherosclerosis and Thrombosis}




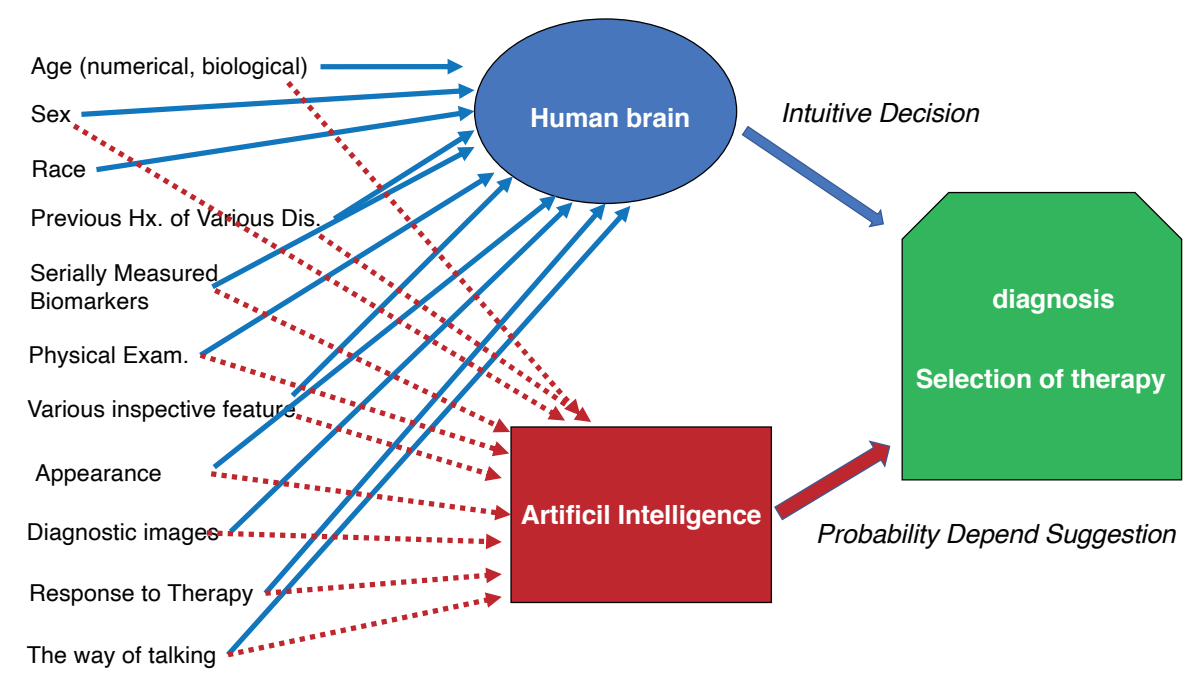

Fig. 1. Human intuition and quantifiable probability calculation by AI

Providers make diagnoses and therapeutic plans considering extensive multi-dimensional data from individual patients in the context of the clinical evidence base. The accuracy of diagnosis and appropriateness of the choice of treatments are difficult to validate, as they are heavily influenced by "intuition" (solid lines). Highly advanced computers are now able to better quantify the relationships between multi-dimensional input and single dimensional outcome of the risks of various clinical events (dotted lines).

accumulated knowledge, and subjective intuition-all of which can be avoided in AI analytic processes. The "intuition-based" decision is the strength of the human brain. But, it is hard to quantify or optimize the accuracy of human "intuition", which is not standardized. Thus, AI has the potential to evolve "expert intuition" into quantifiable science with the potential to improve the quality and accuracy of personalized medicine and individual-based clinical decision making. A few such examples converting "intuition" into more objective, quantitative approaches include: AI-enhanced electrocardiography $(\mathrm{ECG})^{4-7)}$; ultrasonic echocardiography $(\mathrm{UCG})^{8,9)}$; and serial bio-marker analyses ${ }^{10)}$; each of which provide patient-level quantitative $\mathrm{CV}$ disease risk prediction using multi-dimensional input data.

Technology evolution of multi-core graphics processing units (GPUs) has enabled complex AI calculations to be done on personal computers. The widespread investigation into and the use of various AI processes and programs across the CV landscape, analyzing data from huge clinical databases in the era of highly advanced HPC, could ultimately enable providers and patients to use individualized clinical risk prediction to inform clinical decision making-the mission of "precision medicine". Through the application of AI methods, health care providers could potentially provide quantitative estimates of benefits and risks for each clinical decision or therapy considered, complementing but not replacing their "expert intuition", yielding more informed choices and decision making for patients. Shared decision making between providers and patients could be enhanced in the era of advanced AI modeling. It is important to note that the final therapeutic decisions assimilating all such information inputs will still need the contributions of human brains from both patients and providers because the method of AI modeling is a "black box" lacking the common sense of the human brain, and could not easily detect edge cases where AI models fail.

Due to the limitation of the ability of the human brain to completely understand multi-dimensional to multi-dimensional relationships quantitatively, current clinical studies are usually carried out assessing singledimensional outcome data such as the future risk of $\mathrm{CV}$ events. Even in a setting where multiple outcomes such as CV death/MI/stroke, stroke/systemic embolism, serious bleeding, onset of cancer, etc. are considered in a study, these are either treated individually or simply combined to form a single dimensional composite outcome failing to account for whether the individual components of the outcome are interrelated ${ }^{11)}$. Competing risk models have been used to overcome some of these limitations, but it is still challenging to predict events when multiple outcomes are strongly related. Analyses using AI methods are capable of connecting multi-dimensional input to multi-dimensional output.

\section{Advance Publication Journal of Atherosclerosis and Thrombosis}




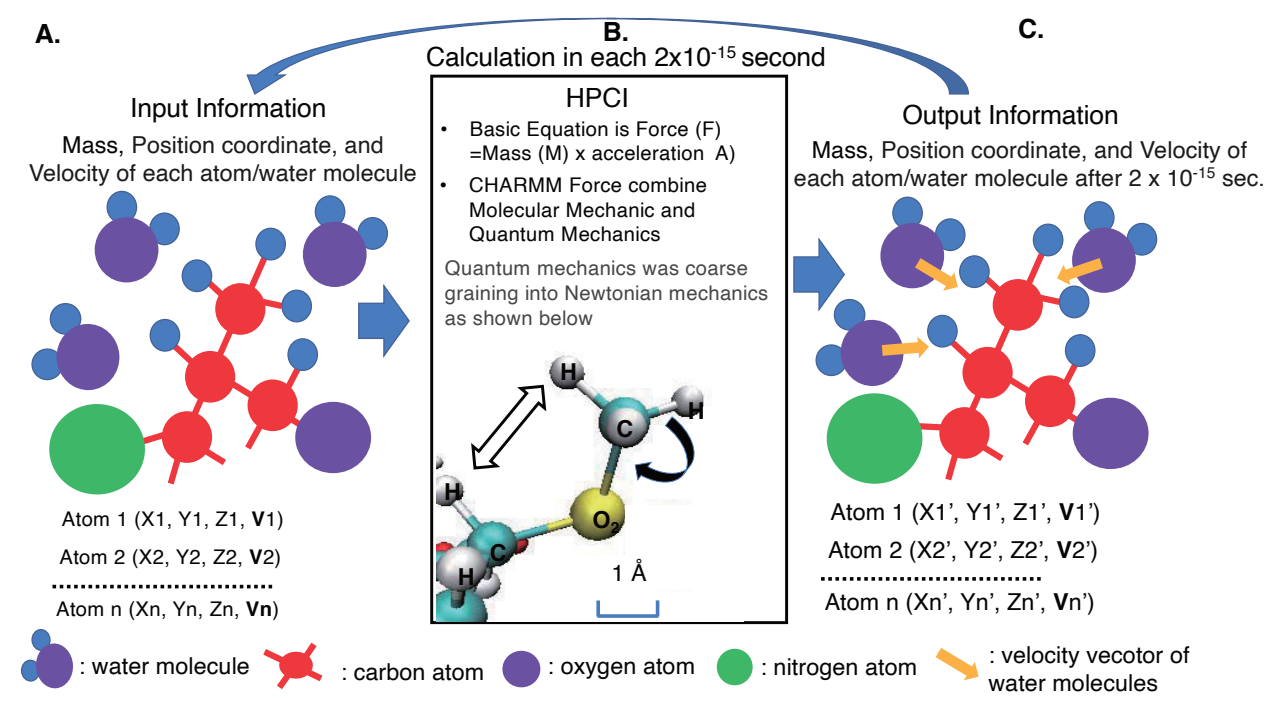

Fig. 2. Prediction of Dynamic Structure and Function of Various Proteins from the Physical Movement of Atoms

The left panel shows the positions of all atoms constructing specific proteins. Each atom is exposed by force generated by the presence of other atoms and water molecules. The physical characteristics of each atom could be express as the vectors constructed from position coordinate and velocity vector such as (X1, Y1, Z1, V1). The position coordinates and vector are changed by the influence of forces applied to them. The HPC enables the calculation of the position coordinates and construction of velocity vectors of all atoms and water molecules within very short periods such as $2 \times 10^{-15}$ second as shown in the right panel. Continuous calculation for $10^{6}$ times enables the prediction of dynamic structural changes of specific proteins.

\section{HPC for Basic Science to Understand the Mechanism of Cardiovascular Diseases}

Beyond prognostic modeling and informing clinical care, the use of AI has the ability to identify otherwise unidentified/unexpected relationships between multi-dimensional data and clinical outcomes, that beyond prognosis, may enlighten/ uncover potential mechanistic pathways of disease via hypothesis generation. However, unraveling association from causality and drilling down into mechanistic pathways triggered by AI results will remain challenging to define pathways and identify potential novel therapeutic targets. The established constructive approach will remain critical for understanding of complex biological phenomena.

HPC technology has the potential to facilitate this constructive approach in CV basic research. In general, complex biological phenomena can be reduced to combinations of simple biological/ chemical/physical laws. In the constructive approach, biological phenomena are attempted to be explained starting from these simple principles. One major success using this approach is the prediction of coronary artery fractional flow reserve by analyzing computer tomography images ${ }^{12)}$, where the basic Navier-Stokes equation is solved discretely by computer simulation on $\mathrm{HPC}^{13}$. Another example of a potentially useful application of HPC, starting from basic/simple constructs, is the prediction of protein interactions that mediate specific biological functions. (Fig.2). A practical example of this is the Chemistry at Harvard Macromolecular Mechanics (CHARMM) HPC project that uses quantum mechanics and Newton's $2^{\text {nd }}$ law to calculate muscle contraction by myosin-actin interactions ${ }^{14)}$, protein structure change caused by a point mutation in patients with pulmonary artery hypertension ${ }^{15}$, prediction of the mechanism of platelet-type von Willebrand disease ${ }^{16,17)}$, and so on. These successes indicate that HPC enables the modeling of complex biological phenomena that influence $\mathrm{CV}$ events from very simple equations, may help understanding them at the theoretical level, and may even identify a new therapeutic target by resolving the dynamic protein structure mediating biological functions.

\section{Conclusion}

The rapid evolution of HPC processes and methods, including AI applications to routine clinical data, has reached the stage to influence the field of clinical CV science. Advanced HPC also plays an important role for understanding the mechanism of cardiology-related events constructed from simple biological/chemical/physical laws and may ultimately

\section{Advance Publication Journal of Atherosclerosis and Thrombosis}


reveal new targets for drug discovery.

\section{Acknowledgements}

Shinya Goto acknowledge a grant-in-aid for MEXT/JSPS KAKENHI 19H03661, AMED grant number A368TS, Bristol-Myers Squibb for independent research support project (33999603) a grant from Nakatani Foundation for Advancement of Measuring Technologies in Biomedical Engineering, and Vehicle Racing Commemorative Foundation (6236).

\section{Disclosures}

Dr. Shinya Goto discloses modest grant support from Sanofi, Pfizer, Bristol Myer Squibb and Ono Pharma. Dr. Shinya Goto acknowledges clinical trial executive committee fees from Janssen. Shinya Goto also acknowledges clinical trial steering committee member fees and consultant fees from Anthos. Dr. Darren McGuire and Dr. Shinichi Goto have nothing to disclose.

\section{References}

1) Schreiber LR and Bluhm H: Toward a silicon-based quantum computer. Science, 2018; 359: 393-394

2) Sravanthi G, Grace B and Kamakshamma V: A review of High Performance Computing. IOSR Journal of Computer Engineering, 2014; 16: 36-43

3) Jaeger H: Artificial intelligence: Deep neural reasoning. Nature, 2016; 538: 467-468

4) Siontis KC, Noseworthy PA, Attia ZI and Friedman PA: Artificial intelligence-enhanced electrocardiography in cardiovascular disease management. Nature Reviews Cardiology, 2021;

5) Goto S, Kimura M, Katsumata Y, Goto S, Kamatani T, Ichihara G, Ko S, Sasaki J, Fukuda $K$ and Sano $M$ : Artificial intelligence to predict needs for urgent revascularization from 12-leads electrocardiography in emergency patients. PLoS One, 2019; 14: e0210103

6) Attia ZI, Kapa S, Lopez-Jimenez F, McKie PM, Ladewig DJ, Satam G, Pellikka PA, Enriquez-Sarano M, Noseworthy PA, Munger TM, Asirvatham SJ, Scott CG, Carter RE and Friedman PA: Screening for cardiac contractile dysfunction using an artificial intelligenceenabled electrocardiogram. Nat Med, 2019; 25: 70-74

7) Feeny AK, Chung MK, Madabhushi A, Attia ZI, Cikes M, Firouznia M, Friedman PA, Kalscheur MM, Kapa S, Narayan SM, Noseworthy PA, Passman RS, Perez MV, Peters NS, Piccini JP, Tarakji KG, Thomas SA, Trayanova NA, Turakhia MP and Wang PJ: Artificial Intelligence and Machine Learning in Arrhythmias and Cardiac Electrophysiology. Circ Arrhythm Electrophysiol, 2020; 13: e007952

8) Goto S, Mahara K, Beussink-Nelson L, Ikura H, Katsumata Y, Endo J, Gaggin HK, Shah SJ, Itabashi Y, MacRae CA and Deo RC: Artificial intelligence-enabled fully automated detection of cardiac amyloidosis using electrocardiograms and echocardiograms. Nat Commun, 2021; 12: 2726

9) Zhang J, Gajjala S, Agrawal P, Tison GH, Hallock LA, Beussink-Nelson L, Lassen MH, Fan E, Aras MA, Jordan C, Fleischmann KE, Melisko M, Qasim A, Shah SJ, Bajcsy R and Deo RC: Fully Automated Echocardiogram Interpretation in Clinical Practice. Circulation, 2018; 138: $1623-1635$

10) Goto S, Goto S, Pieper KS, Bassand JP, Camm AJ, Fitzmaurice DA, Goldhaber SZ, Haas S, Parkhomenko A, Oto A, Misselwitz F, Turpie AGG, Verheugt FWA, Fox KAA, Gersh BJ, Kakkar AK and Investigators G-A: New AI Prediction Model Using Serial PT-INR Measurements in AF Patients on VKAs: GARFIELD-AF. Eur Heart J Cardiovasc Pharmacother, 2019;

11) Steg PG and Bhatt DL: Is There Really a Benefit to Net Clinical Benefit in Testing Antithrombotics? Circulation, 2018; 137: 1429-1431

12) Curzen NP, Nolan J, Zaman AG, Nørgaard BL and Rajani R: Does the routine availability of CT-derived FFR influence management of patients with stable chest pain compared to CT angiography alone? The FFRCT RIPCORD study. JACC: Cardiovascular Imaging, 2016; 9: 1188-1194

13) Vignon-Clementel IE, Figueroa CA, Jansen $\mathrm{KE}$ and Taylor CA: Outflow boundary conditions for threedimensional finite element modeling of blood flow and pressure in arteries. Computer methods in applied mechanics and engineering, 2006; 195: 3776-3796

14) Li $\mathrm{M}$ and Zheng W: All-Atom Molecular Dynamics Simulations of Actin-Myosin Interactions: A Comparative Study of Cardiac $\alpha$ Myosin, $\beta$ Myosin, and Fast Skeletal Muscle Myosin. Biochemistry, 2013; 52: 8393-8405

15) Shinya Y, Hiraide T, Momoi M, Goto S, Suzuki H, Katsumata Y, Kurebayashi Y, Endo J, Sano M, Fukuda K, Kosaki K and Kataoka M: TNFRSF13B c.226G $>$ A (p.Gly76Ser) as a Novel Causative Mutation for Pulmonary Arterial Hypertension. J Am Heart Assoc, 2021; 10: e019245

16) Goto S, Oka H, Ayabe K, Yabushita H, Nakayama M, Hasebe T, Yokota H, Takagi S, Sano M, Tomita A and Goto S: Prediction of binding characteristics between von Willebrand factor and platelet glycoprotein Ibalpha with various mutations by molecular dynamic simulation. Thromb Res, 2019; 184: 129-135

17) Shiozaki S, Takagi S and Goto S: Prediction of Molecular Interaction between Platelet Glycoprotein $\mathrm{Ib} \alpha$ and von Willebrand Factor using Molecular Dynamics Simulations. J Atheroscler Thromb, 2016; 23: 455-464 